LA-UR. $96-1554 \quad$ CONF- $961004-1$

TITLE:

AUTHOR(S):

SUBMITTED TO:

\section{APPROXIMATE OPTION PRICING}

Prasad Chalasani

Somesh Jha

Isaac Saias

\title{
MASTER
}

By acceptance of this article, the publisher recognizes that the U.S. Government retains a nonexclusive royalty-free license to publish or reproduce the published form of this contribution or to allow others to do so, for U.S. Government purposes.
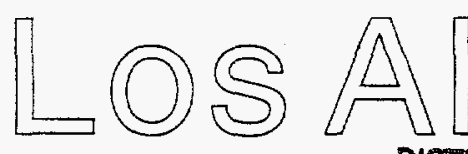


\section{DISCLAIMER}

Portions of this document may be illegible in electronic image products. Images are produced from the best available original document. 



\title{
Approximate Option Pricing
}

\author{
PRASAD CHALASANI \\ Los Alamos National Laboratory \\ chal@lanl.gov
}

\author{
SOMESH JHA \\ Carnegie Mellon University \\ sjha@cs.cmu.edu
}

\author{
ISAAC SAIAS \\ Los Alamos National Laboratory \\ isaac@lanl.gov
}

April 8, 1996

\begin{abstract}
As increasingly large volumes of sophisticated options are traded in world financial markets, determining a "tair" price for these options has become an important and difficult computational problem. Many valuation codes use the binomial pricing model, in which the stock price is driven by a random walk. In this model, the value of an $n$-period option on a stock is the expected time-discounted value of the future cash flow on an $n$-period stock price path. Path-dependent options are particularly difficult to value since the future cash flow depends on the entire stock price path rather than on just the final stock price. Currently such options are approximately priced by Monte Carlo methods with error bounds that hold only with high probability and which are reduced by increasing the number of simulation runs.

In this paper we show that pricing an arbitrary path-dependent option is \#-P hard. We show that certain types of path-dependent options can be valued exactly in polynomial time. Asian options are pathdependent options that are particularly hard to price. and for these we design deterministic polynomialtime approximate algorithms. We show that the value of a perpetual American put option (which can be computed in constant time) is in many cases a good approximation to the value of an otherwise identical $n$-period American put option. In contrast to Monte Carlo methods, our algorithms have guaranteed error bounds that are polynomially small (and in some cases exponentially small) in the maturity $n$. For the error analysis we derive large-deviation results for random walks that may be of independent interest.
\end{abstract}

\section{DISCLAIMER}

This report was prepared as an account of work sponsored by an agency of the United States Government. Neither the United States Government nor any agency thereof, nor any of their employees, makes any warranty, express or implied, or assumes any legal liability or responsibility for the accuracy, completeness, or usefulness of any information, apparatus, product, or process disclosed, or represents that its use would not infringe privately owned rights. Reference herein to any specific commercial product, process, or service by trade name, trademark, manufacturer, or otherwise does not necessarily constitute or imply its endorsement, recommendation, or favoring by the United States Government or any agency thereof. The views and opinions of authors expressed herein do not necessarily state or reflect those of the United States Government or any agency thereof. 


\section{Introduction}

Over the last deciade or so. sophisticated financial instruments called derivative securities [9, 16] have become increasingly important in world financial markets. These are securities whose value depends on the values of more basic underlying variables. For instance a stock option is a derivative security whose value is contingent on the price of a stock. The trade in options, futures and other types of derivative securities often has a value exceeding that of the underlying asset [27]. Hedgers find it advantageous to trade in a derivative security on an asset rather than in the asset itself. to reduce the risk associated with the price of the asset. Also, speculators trade in options on stocks to get extra leverage from a favorable movement of the stock price.

Of course, a price must be paid to own a derivative security, and a central problem is the one of determining a "fair" price. An option is priced, or "valued", by assuming (a) some model of the price behavior of the underlying asset (e.g., a stock), and (b) a pricing theory. In a landmark paper, Black and Scholes [2] introduced a continuous-time model for option valuation that underlies most pricing methods in use today. Their model is based on Arbitrage Pricing Theory $[9,16]$. The model assumes that the asset price is driven by a Brownian motion, and specifies a stochastic differential equation that the option value must satisfy.

For many complex options, such as Asian Options and (American) Lookback options, the Black-Scholes differential equation has no known closed form solution, so numerical approximations are used. In Monte Carlo methods $[4,21,22]$ one runs several continuous-time simulations of the Black-Scholes model to estimate the option price - which is the time-discounted expectation of the future cash flow. This approach is justified by the law of large numbers. In finite difference methods $[7,17,27]$ the underlying stochastic differential equation is discretized and solved iteratively. The error bound typically guaranteed by Monte Carlo methods is $O(\sigma / \sqrt{\mathrm{V}})$, where $N$ is the number of simulation runs, and $\sigma$ is the standard deviation of the future cash flows [21]. It should be noted that this bound only holds with "high" probability, is expressed in terms of the extrinsic parameter $N$, and depends on the underlying dynamic only through $\sigma$. On the other hand, approximations based on finite-difference methods usually lack a precise quantification of the error term (see [24]).

In contrast to the above methods, the widely-used binomial pricing model $[8,16]$ is based on a simpler discrete-time process. The mathematical justification of this model is that the standard symmetric random walk, appropriately scaled, converges to the Brownian motion. As in the continuous models, the price of an $n$-period option is the time-discounted expected value of the future cash-flows over $n$ periods. Even under this model, path-dependent options [18] such as Asians and Lookbacks are particularly difficult to value: for such options, the future cash flows depend on the entire stock price path rather than on just the final stock price, and there are $2^{n}$ possible paths.

In this paper, we study the option pricing problem from the rigorous perspective of computational complexity and approximation algorithms. We assume the binomial model throughout. We show that the problem of pricing arbitrary path-dependent options is \#-P hard. For certain path-dependent options we show polynomial-time exact pricing algorithms. For the notoriously hard Asian option pricing problem, we design deterministic polynomial-time (in $n$ ) approximation algorithms. In contrast to the Monte Carlo methods. our error bounds are expressed in terms of intrinsic parameters such as the maturity $n$ of the option: in fact they are polynomially and in some cases exponentially small in $n$. In some cases our algorithms run in time independent of $n$. We also show that in some cases the price of an American option can be approximated well by that of an otherwise equivalent perpetual option, whose value is $O(1)$-time computable. For the error analysis we prove several large-deviation results on random walks. We thus hope to demonstrate that the field of derivative securities is a rich source of opportunities for computer science research.

For more details on option pricing and the Arbitrage Pricing Theory, the interested reader is referred to the Hull's [16] excellent introductory text. However the present paper defines all the relevant concepts needed, and will suffice to understand the computational problems involved. Section 1.1 describes the bino- 
mial model for stock prices. Section 1.2 defines the options considered in this paper, and Section 1.3 descrihes the pricing formulas and the specific results in the paper. The remaining sections contain our results.

\subsection{The binomial model for stock prices}

To keep the wording simple. we only consider options on stocks. The notation described in this section will be used throughout the paper. For easy reference, at the end of the Appendix we include a summary of the notation used in this paper. The binomial model for the price of the stock underlying an $n$-time-period ( $n \geq 1$ ) option is the following. The model is parametrized by the constants p.q.u.r. These parameters are determined independently, and we will assume they are known. $n$ is the (possibly infinite) number of time periods up to the expiration of the option. where time 0 is the initial time, i.e., the time at which one wants to price the option. The trading dates are times $0.1, \ldots n$. The stock price at time $k$ is denoted $S_{k}$. The initial stock price $S_{0}$ is assumed to be non-random. $u$ is the up-factor, $p$ is the up-tick probability, $r$ is the risk-free interest rate. At each time step, with probability $p$ the stock price goes up by a factor $u$, and with probability $q=1-p$ the price goes down by a factor $1 / u$. The parameters $u . p . q, r$ satisfy (see [16]):

$$
\begin{gathered}
u>1 . \quad 1 / u<1+r<u \\
0<p<1, \quad p+q=1 . \\
p=\frac{1+r-1 / u}{u-1 / u} . \quad \text { or equivalently. } \quad p u+q / u=1+r .
\end{gathered}
$$

We now formalize the model. It will be convenient to visualize a sequence of $n$ independent coin-tosses $\nu=\left(\omega_{1}, \omega_{2} \ldots \omega_{n}\right)$, where each $\omega_{i} \leqslant\{H, T\} ;$ an $H$ corresponds to an "up-tick" of the stock price, and a $T$ corresponds to a "down-tick". A particular sequence of coin-tosses $\omega$ will be referred to as a path. The sample space $\Omega$ is the set of all possible coin-toss sequences $\omega$. We define the random variables $X_{1}, X_{2} \ldots \ldots X_{\text {ri }}$ where for any $\nu \in \Omega$,

$$
X_{i}(\omega)= \begin{cases}+1 & \text { if } \omega_{i}=H \\ -1 & \text { otherwise }\end{cases}
$$

We define the probability measure $\mathbf{P}$ on $\Omega$ to be the unique measure for which the random variables $X_{i}, i=$ $1.2 \ldots n$, are independent, identically distributed (iid) with $\mathbf{P}\left[X_{i}=1\right]=p$ and $\mathbf{P}\left[X_{i}=-1\right]=q=1-p$. We will refer to the sequence of random variables $\left\{X_{i}\right\}_{i=1}^{n}$ with the above distribution as the random walk with drift $p$. Then the stock price $S_{k}, k \geq 0$, is a random variable that satisfies

$$
S_{k+1}=S_{k} u^{X_{k+1}} \text {. }
$$

We also define $Y_{0}=0$, and for $k \geq 1, Y_{k}=\sum_{i=1}^{k} X_{i}$, and $T_{k}=\sum_{i=1}^{k} S_{i}$. Thus we can write for $k \geq 0$, $S_{k}=S_{0} u u^{\xi_{k}}$.

For any integer $k \geq 0$, for any random variable $Z$, the conditional expectation $\mathbf{E}\left[Z \mid X_{1}, X_{2} \ldots \ldots \dot{X}_{k}\right]$ of $Z$ given the first $k$ coin tosses will be denoted $\mathbf{E}\left[Z \mid \mathcal{F}_{k}\right]$. In particular $\mathbf{E}\left[Z \mid \mathcal{F}_{0}\right] \triangleq \mathbf{E}[Z]$. For any integer $k \geq 1$, a random variable $Z$ is $\mathcal{F}_{k}$-measurable if it depends only on the first $k$ coin tosses, i.e., on $X_{1}, X_{2}, \ldots, X_{k}$. An $\mathcal{F}_{0}$-measurable random variable is non-random.

It is common to refer to a sequence of random variables as a process. In particular, $\left\{S_{k}\right\}_{k=0}^{n}$ is the stock price process. A process $\left\{Z_{k}\right\}_{k=0}^{n}$ such that each $Y_{k}$ is $\mathcal{F}_{k}$-measurable, is said to be adapted. Thus the stock price process is adapted. For any process $\left\{Z_{k}\right\}_{k=0}^{n}$ we write $\bar{Z}_{k}$ to denote $\max _{0 \leq i \leq k} Z_{i}$. Similarly, $\underline{Z}_{k} \triangleq \min _{0 \leq i \leq k} Z_{i}$ 


\subsection{Options}

There are two basic types of options. A call option on a stock is a contract that gives the holder the right to buy the underlying stock by a certain date, tor a certain price. A put option gives the holder the right 1 , sell the underlying stock by a certain date for a certain price. The price in the contract is known as the strike price, and is denoted by $k$. The date in the contract is known as the exercise date, or expiration date. Recall that $n$ denotes the number of time perinds until the expiration of the option. The holder of the option must pay a certain price, called the option price to the issuer of the option. The option pricing problem is to determine the "fair" price to pay for an option. This will become clearer later. An American option can be exercised at any time up to the expiration date. European options can only be exercised on the expiration date itself. It is important to note that an option contract merely gives the holder the right to exercise: the holder need not exercise it.

The payoff $G_{k}$ from an option (for the holder) at time $k$ is 0 if it cannot be exercised at time $k$. Otherwise $G_{k}$ is the maximum of 0 and the profit that can be realized by exercising the option at time $k$. This protit ignores the price paid by the buyer for the option. For instance consider an American Call option. If $S_{k}>h$. the holder can exercise the option at time $k$ by buying the stock at $K$ and realize a profit of $S_{k}-k$ by selling the stock in the market at $S_{k}$. If $S_{k} \leq K$, no positive profit can be made by exercising. Thus, for an American call, the payoff is the random variable

$$
G_{k}=\left(S_{k}-K\right)^{+}, k=0,1.2 \ldots, n . \quad \text { (Payoff for American call) }
$$

where for any $x \in \mathbb{R}, x^{+}=\max \{x, 0\}$. Similarly for an American put,

$$
G_{k}=\left(K-S_{k}\right)^{+}, k=0,1,2 \ldots, n . \quad \text { (Payoff for American put) }
$$

The payoff functions for the European options are the same as for their American counterparts, except that exercise is only allowed at time $k=n$, so $G_{k}=0$ for all $k<n$.

In the case of simple calls and puts, the payoff at any time depends only on the prevailing stock price, i.e.. $G_{k}=g\left(S_{k}\right)$ for some function $g$. Such options are said to be Markovian, or path-independent. However there are many options that are path-dependent $[13,16,18]$. One class of such options we consider in this paper are Asian options. An (European-style) Asian call option is one that can be exercised only at time $n$, and whose payotf $G_{n}$ is given by

$$
G_{n}=\left(\mu_{n}-K\right)^{+}, \quad \text { (Payoff for Asian call) }
$$

where $\mu_{n}$ is the average stock price from time 1 to time $n: \mu_{n}=T_{n} / n$. We do not include $S_{0}$ in the computation of the average only for notational convenience: since $S_{0}$ is a fixed constant, this does not affect our results.

Similarly, a (European-style) Asian put has payoff

$$
G_{n}=\left(K-\mu_{n}\right)^{+} . \quad \text { (Payoff for Asian put) }
$$

Asian options are of obvious appeal to a company which must buy a commodity at a fixed time each year. yet has to sell it regularly throughout the year [27]. These options allow investors to eliminate losses from movements in an underlying asset without the need for continuous rehedging. Such options are commonly used for currencies [27], interest-rates and commodities such as crude oil [14].

We consider two other path-dependent option payoffs in the paper: (Let $1_{A}$ denote the indicator function for any subset $A \subseteq \Omega$.)

$$
G_{k}=\left\{\begin{array}{lr}
\left(\bar{S}_{k}-K\right)^{+}, k \geq 0 & \text { (Lookback option) } \\
\overline{1}_{\bar{S}_{k} \geq B}\left(S_{k}-k\right)^{+}, k \geq 0 & \text { (Knock-in barrier option) }
\end{array}\right.
$$


We also consider the American perpetual put (APP) option, which has an associated strike price $h$ just like an ordinary American put. except that there is no expiration date. The payoff $G_{k}$ for an APP is theretore given by

$$
C_{i}=\left(K-S_{k}\right)^{+} . \quad k \geq 0 . \quad \text { (Payotf for American perpetual put) }
$$

\subsection{Pricing formulas, and results in the paper}

Since a European option can be viewed as an American option with payolf $G_{k}=0$ for all $k<n$. pricing formulas for American options apply equally well to European options. However, the formulas for European options are somewhat simpler and we describe them tirst.

For European-style options with payoff $G_{n}$, the value of the option at time $k$ is defined by

$$
V_{k}=(1+r)^{k} \mathbf{E}\left[(1+r)^{-n} G_{n} \mid \mathcal{F}_{k}\right], \quad k=0.1 \ldots n .
$$

which is the expected payoff at expiration, discounted by the risk-free interest rate over $n-k$ periods. In particular we have $V_{n}=G_{n}$. We refer to the time-0 value $V_{0}$ as simply "the value" of the option, and denote it by $V$ :

$$
r \triangleq l_{0}=(1+r)^{-n} \mathbf{E} G_{n}
$$

The pricing problem, which this paper deals with. consists of evaluating the formula (E) for the value $V$ of an option. We show in Section 2 that this problem is \#-P hard for an arbitrary (polynomially-specified) path-dependent European option. It is easy to see that ordinary European calls and puts can easily be valued in $O(n)$ time. However, the valuation of Asian calls and puts is a well-known hard problem in finance and much research has been directed at this problem [3, 11, 25, 27, 28]. All known valuation methods for these options either use some form of Monte Carlo estimation or use analytic approximations with no error analysis. For instance, Turnbull and Wakeman [25] have proposed an analytic approximation for Asian options. but provide no error analysis: they only experimentally test the accuracy of their approximation against Monte Carlo estimates. In Section 4 we develop deterministic polynomial-time approximation algorithms for the value $V$ of Asian options, along with error bounds. For the error analysis we show several large-deviation results for random walks that may be of independent interest.

To define the value of an American option, we need to use the notion of a stopping time (see the Appendix). For an American option with payoff functions $\left\{G_{k}\right\}_{k=0}^{n}$ (where $n$ can be infinite), the value at time $k$ is given by

$$
V_{k}=(1+r)^{k} \max _{\tau \in \mathcal{T}_{k}} \mathrm{E}\left[(1+r)^{-\tau} G_{\tau} \mid \mathcal{F}_{k}\right]
$$

where $\mathcal{T}_{k}$ is the class of stopping times $\tau$ satisfying $k \leq \tau \leq n$ almost surely. In particular, the value of the option at time 0 (which we simply refer to as "the value" $V$ ) is

$$
V=V_{0}=\max _{\tau \in T_{0}} \mathbf{E}\left[(1+r)^{-\tau} G_{\tau}\right]
$$

The value $I$ of an American perpetual put (APP) does not involve $n$, and it can be computed in $O(1)$ time in closed form. It is natural therefore to use this value to estimate the value of an otherwise identical $n$-period American put. In Section 5 we investigate the error of this estimate.

For a Markovian option with payoff $G_{k}=g\left(S_{k}\right)$, the definition (4) implies that $V_{k}=v_{k}\left(S_{k}\right)$ for some function $v_{k}$, where $v_{k}$ satisfies:

$$
\begin{aligned}
& r_{n}\left(S_{n}\right)=g\left(S_{n}\right) \quad \text { (only for options with finite } n \text { ) } \\
& v_{k}\left(S_{k}\right)=\max \left\{g\left(S_{k}\right) \cdot \frac{1}{1+r}\left(p v_{k+1}^{\prime}\left(u S_{k}\right)+q v_{k+1}\left(\frac{S_{k}}{u}\right)\right)\right\} . \quad k=0,1 \ldots
\end{aligned}
$$


The backward-recursion equation (5) allows $l^{\prime}$ to be computed by dynamic programming in $O\left(n^{2}\right)$ timc. since there are only $k+1$ possible different values for $S_{k}$. In Section 3 we extend this approach to certain path-dependent options (such as the Lookback and Knock-in barrier options) whose payoff can be expressed as a function of a Markov process different from the stock price process $\left\{S_{k}\right\}$.

\section{Pricing an arbitrary European option is \#P-hard}

Consider a European option with an arbitrary path-dependent payoff function $G_{n}$. We will restrict our attention to payoff functions $G_{n}$ that can be specified in space polynomial in $n$. We then wish to evaluate $V \leqq V_{i}$. We show that evaluating $V$ is \#P-hard.

Theorem 1 The problem of pricing a European option with polynomially-specified payoff function $C_{t:}, i s$ \#P-hard.

Proof: It is well-known that the following counting problem is \#P-complete: Given a graph.$J$ with edge-set $E=\left\{\epsilon_{1}, \epsilon_{2}, \ldots \epsilon_{n}\right\}$. count the number $M(J)$ of perfect matchings in $J$. We reduce this problem to the pricing problem. We define a (path-dependent) European option with expiration time $n$ whose payoff $G_{r_{i}}$ is given by:

$$
G_{n}\left(\nu^{\prime}\right)= \begin{cases}1 & \text { if }\left\{\epsilon_{i}: \omega_{i}=H\right\} \text { is a perfect matching of } J \\ 0 & \text { otherwise. }\end{cases}
$$

Next we choose $r$ and $u$ so that $1+r=(u+1 / u) / 2$, so that $p=q=\frac{1}{2}$. Thus every path $\omega$ has probability $\mathbf{P}(\omega)=\left(\frac{1}{2}\right)^{n}$. Clearly, from Eq. (E) the value of this option is

$$
V=(1+r)^{-n} \sum_{\omega \in\{0.1\}^{n}} \mathbf{P}(\omega) G_{n}(\omega)=(1+r)^{-n}\left(\frac{1}{2}\right)^{n} \sum_{\omega \in\{0.1\}^{n}} G_{n}(\omega)=(1+r)^{-n}\left(\frac{1}{2}\right)^{n} . M(J) .
$$

Thus if we can compute $V$ exactly in polynomial time, then we can also compute $M(J)$ in polynomial time.

\section{Exact pricing of some path-dependent options}

We saw in Section 1.3 that the value $V$ of a Markovian option can be computed in $O\left(n^{2}\right)$ time by dynamic programming, using the backward recursion formula (5). We generalize this dynamic programming approach to certain path-dependent options, such as the Lookback option, and the Knock-in barrier option. The main observation is that the backward-recursion formula (5) depends only on the fact that the stock price process $\left\{S_{k}\right\}$ is a Markov process, i.e., for $k \geq 0$, if $h$ is any (Borel-measurable) function, then

$$
\mathbf{E}\left[h\left(S_{k+1}, S_{k+2}, \ldots, S_{n}\right) \mid \mathcal{F}_{k}\right]=\mathbf{E}\left[h\left(S_{k+1}, S_{k+2} \ldots, S_{n}\right) \mid S_{k}\right] .
$$

Therefore, we have the following theorem:

Theorem 2 Consider an American option with payoff process $\left\{G_{k}\right\}_{k=0}^{n}$ where $G_{k}=g\left(C_{k}\right)$ where $C_{k}$ is an adapted Markov process such that for each $k$, the number of different possible values of $C_{k}$ is a polynomial in $n$, and the set of possible values of $C_{k}$ is known. Then the value $V$ for this option can be computed in time polynomial in $n$ using dynamic programming.

For instance, it is not hard to show that the process $C_{k} \triangleq\left(S_{k} . \bar{S}_{k}\right), k=0,1, \ldots n$ is a Markov process. Moreover, for each $k$, there are at most $(k+1)^{2}$ possible combinations of values $\left(S_{k}, \vec{S}_{k}\right)$. Both the Lookback option and the Knock-in barrier option (see Section 1.2) have payolf functions $G_{k}$ expressible as function.s of $\left(S_{k}, \bar{S}_{k}\right)$, so they can be priced in $O\left(n^{3}\right)$ time by dynamic programming. 


\section{Approximate Pricing of Asian Options}

We wish to approximate the value $V$ for Asian calls and puts given by the formulae in Section 1.3. Computing $\mathbf{E}\left(\mu_{r}-h^{+}\right.$(or $\left.\mathbf{E}\left(h^{-}-\mu_{n}\right)^{+}\right)$exactly is known to be a hard problem in tinance (the exact computational complexity of this problem is not known). However, we can compute $\mathrm{E} \mu_{r}$ in closed form:

$$
\begin{aligned}
\mathbf{E} \mu_{n} & =\frac{S_{0}}{n}\left[\mathbf{E}\left(u^{x_{1}}\right)+\mathbf{E}\left(u^{x_{1}+x_{2}}\right)+\ldots+\mathbf{E}\left(u^{\left.\left.x_{1}+x_{2}+\ldots+x_{n}\right)\right]}\right.\right. \\
& =\frac{S_{0}}{n}\left[\alpha+\alpha^{2}+\ldots+a^{n}\right]=\frac{S_{0} n}{n} \frac{a^{r_{n}}-1}{a-1}=\frac{S_{n}(1+r)\left[(1+r)^{n_{n}}-1\right]}{n r} .
\end{aligned}
$$

where $\alpha \triangleq \mathbf{E}\left(u^{X_{1}}\right)=p u+q(1 / u)=1+r$. It is not hard to see that the quantities $(1+r)^{-n}\left[\mathbf{E}_{\mu_{r}}-h_{1}{ }^{+}\right.$ and $(1+r)^{-n}\left[K-\mathrm{E} \mu_{n}\right]^{+}$respectively approximate the value $V$ of an Asian call and an Asian put to within $(1+r)^{-n} K$.

We now describe polynomial-time approximation algorithms that are significantly better. The error analysis of these algorithms is based on certain large-deviation results on random-walks that we derive in Section 4.1. We use the notation $3=|2 p-1|$ since this value appears frequently in the error bounds. In the following description, we use the symbols $\mathbf{P}_{\ell}(c, n) . \mathbf{P}_{\varepsilon}(n), \mathbf{P}_{g}(n)$ (corresponding to the cases $p$ less than, equal to and greater than $\frac{1}{2}$ respectively) to stand for different probabilities that will be determined in the next section. In most cases we can express the asymptotic difference between the exact value and our approximation in the form $K(1+r)^{-n} O(f(n))$, where we treat the parameters $S_{0}, u, \beta, r$ as constants.

For $p>\frac{1}{2}$, we show (Theorem 3 , Corollary 4 ) that with probability at least $1-\mathbf{P}_{3}(n)$, all stock prices after $S_{n / 2}$ are at least $2 K$, so that $\mu_{n} \geq K$. For an Asian call, this means that with probability at least $1-\mathbf{P}_{3}(n)$. $\left(\mu_{n}-K\right)^{+}=\left(\mu_{n}-K\right)$, so we approximate

$$
\mathrm{V} \triangleq(1+r)^{-n} \mathbf{E}\left(\mu_{n}-K\right)^{+} \simeq(1+r)^{-n}\left(\mathbf{E} \mu_{n}-K\right)^{+} . \quad \text { (Asian call approx., } p>\frac{1}{2} \text { ) }
$$

When $\left(\mu_{n}-K\right)^{+}$exceeds $\left(\mu_{n}-K\right)$ (which occurs with probability at most $\left.\mathbf{P}_{g}(n)\right)$, the difference between these quantities is at most $K$, so that the error in this constant-time approximation is at most

$$
K^{-}(1+r)^{-n} \mathbf{P}_{y}(n)=K(1+r)^{-n} O\left(1 / e^{3^{2} n / 4}\right) . \quad \text { (Asian call error, } p>\frac{1}{2} \text { ) }
$$

since, as we will show, $\mathbf{P}_{g}(n)=O\left(1 / e^{\beta^{2} n / 4}\right)$. On the other hand, for an Asian put. the above results imply that with probability at least $1-\mathbf{P}_{g}(n),\left(K-\mu_{n}\right)^{+}=0$, so that we can upper bound

$V \triangleq(1+r)^{-n} \mathbf{E}\left(K-\mu_{n}\right)^{+} \leq K(1+r)^{-n} \mathbf{P}_{g}(n)=K(1+r)^{-n} O\left(1 / \epsilon^{3^{2} n / 4}\right) \quad$ (Asian put bound, $\left.p>\frac{1}{2}\right)$,

and use half of this bound as a constant-time approximation:

For $p=\frac{1}{2}$, we show in Theorem 5 and Corollary 6 that with probability at least $1-\mathbf{P}_{s}(n)$, some stock price before time $n$ is at least $n K$, so that the average stock price $\mu_{n}$ is at least $K$. Therefore by the same reasoning as above for an Asian call, the approximation $(1+r)^{-n}\left(\mathbf{E} \mu_{n}-K\right)^{+}$has error at most $K(1+r)^{-n} \mathbf{P}_{\varepsilon}(n)$ where $\mathbf{P}_{e}(n)=O\left(\frac{\log n}{\sqrt{n}}\right)$. As in the case $p>\frac{1}{2}$, we can show that the value $V$ of an Asian put does not exceed $K(1+r)^{-n} \mathbf{P}_{e}(n)$.

For $p<\frac{1}{2}$, Theorem 3 and Corollary 4 establish that for any $c>0$, with probability at least $1-\mathbf{P}_{\ell}(c . n)$, all stock prices after $m=O(c \log n)$ steps are at most $S_{0} / n$. This means that the error in approximating $\mu_{n}=T_{n} / n$ by $T_{m} / n$ is at most $S_{0} / n$. Thus, for an Asian call we can approximate

$$
V \triangleq(1+r)^{-n} \mathbf{E}\left(\mu_{n}-K\right)^{+} \simeq(1+r)^{-n} \mathbf{E}\left(T_{m} / n-K\right)^{+} \quad \text { (Asian call approx., } p<\frac{1}{\underline{2}} \text { ), }
$$


which can be computed in time $O\left(2^{m}\right)=n^{O(:)}$ since we need only consider coin-toss sequences of length $m$. When $\left(T_{n}-T_{m i}\right)>S_{0}$ (which occurs with probability at most $\mathbf{P}_{1}(r n),\left(\mu_{r}-k\right)^{+}$exceeds $\left(T_{m i} n-h^{-}\right.$

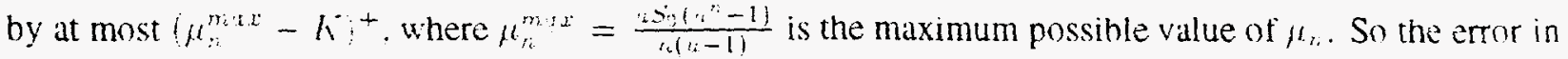
the above approximation is at most

$$
(1+n)^{-n}\left[\frac{S_{0}}{n}+\mathbf{P}_{i}(r \cdot n)\left(\mu_{n i}^{m x}-K^{+}\right)\right] . \quad\left(\text { Asian call error, } p<\frac{1}{2}\right)
$$

Similarly, for an Asian put we use the $n^{O(C)}$-time computable approximation

$$
\mathbf{E}\left(K-T_{m} / n\right)^{+} . \quad\left(\text { Asian put approx, } p<\frac{1}{2}\right)
$$

In this case, the difference between $\left(K-\mu_{n}\right)^{+}$and $\left(K-T_{n} / n\right)^{+}$cannot exceed $K$, so that the error in the approximation is at most

$$
(1+r)^{-n}\left[\frac{S_{u}}{n}+h P_{t}(c . n)\right]
$$

As we will show in the next section, $\mathbf{P}_{\ell}(c . n)=2 /\left(3^{2} n^{c}\right)$, and it can be worked out that with $c=2, \frac{s_{n}}{r_{0}}>$ $K \mathbf{P}_{\ell}(c, n)$. Thus with an $n^{O(1)}$ running time we can achieve an error bound of

$$
\left.(1+r)^{-n} 2 S_{0} / n . \quad \text { (Asian put error, } p<\frac{1}{2}\right)
$$

In the next subsection we derive the large-deviation results that we assumed above. In Appendix A. 3 we describe an algorithm that performs better in practice than the ones we described above. We leave the error analysis of that algorithm as an open problem.

\subsection{Large-deviation results}

We first show the following fact about a drifted random walk. We use the notation for random walks from Section 1.1. In particular recall that $Y_{k}=\sum_{i=1}^{k} X_{i}$ is the $k^{\prime}$ th partial sum of the random walk, and that $T_{k}=\sum_{i=1}^{k} S_{i}$.

Theorem 3 (Drifted Random Walk) Consider the random walk with drift $p$ where $p \neq \frac{1}{2}$. Let $a \in(0.3 n / 2)$. and $m \in(2 a / \beta, n)$. Then with probability at least $1-\frac{2}{\beta^{2}} \exp \left\{3 a-\beta^{2} m / 2\right\}$. for every integer $k \in[m . n]$ :

$$
Y_{k} \geq a \text { if } p>\frac{1}{2} . \quad \text { and } Y_{k} \leq-a \text { if } p<\frac{1}{2}
$$

Proof: The proof. given in Appendix A.2, uses Hoeffding bounds.

Corollary 4 (Average Stock Price in a Drifted Binomial Model) Consider the binomial stock price process $\left\{S_{k}\right\}_{k=0}^{n}$ with $p \neq \frac{1}{2}$. Suppose $K$ is the strike price of an Asian option (call or put). Let

$$
\begin{gathered}
\mathbf{P}_{3}(n) \triangleq \frac{2}{\beta^{2}}\left(\frac{2 K}{S_{0}}\right)^{\frac{3}{\ln u}} \epsilon^{-\beta^{2} n / 4}, \\
\mathbf{P}_{\ell}(c, n) \triangleq \frac{2}{3^{2}} \frac{1}{n^{2}} \quad \text { (c is any positive constant), } m \triangleq\left(\frac{2 c}{\beta^{2}}+\frac{2}{3 \ln u}\right) \ln n .
\end{gathered}
$$

Then: 
1. If $p>\frac{1}{2}$ and if $n>\frac{2}{5} \log _{u}\left(2 K / S_{0}\right)$ then with probability at least $1-\mathbf{P}_{3}(n)$. evern stock price s. for $i \geq n / 2$ is at least $2 K$, and in particular $\mu_{r_{2}} \geq k$.

2. If $p<\frac{1}{2}$. then with probability at least $1-\mathbf{P}_{i}(\therefore n)$, ever stock price $S_{i}$ for $i \geq m$ is at most $S_{11} / n$ and in particular $\mu_{n}-\frac{T_{n}}{n} \leq \frac{S_{n}}{n}$.

Proof: See the Appendix.

For the case $p=\frac{1}{2}$ we would like to show, as in Corollary 4, that with high probability the stock prices $S_{i}$ are all "large" (e.g., at least $2 K$ ) after say $n / 2$ steps. That argument rests on the fact (Theorem 3 ; that with high probability all partial sums in a random walk after a certain point are "large". However the proof of Theorem 3 does not work with $p=\frac{1}{2}$. Instead, we show that with high probability at some time the stock price is at least $n K$, so that the average is at least $K$. For this we use the Berry-Essen Theorem and the Reflection Principle, which we quote in the Appendix.

We first show a large-deviation result for the maximum partial sum of an undrifted random walk.

Theorem 5 (Undrifted random walk) Consider the random walk with $p=\frac{1}{2}$. Recall that $\bar{Y}_{n} \triangleq$ max $_{0 \leq i \leq n} Y_{i}$. Then for any $a>0$.

$$
\mathbf{P}\left[\bar{Y}_{n} \leq\lfloor a\rfloor\right\rfloor \leq \sqrt{2 / \pi} \frac{a}{\sqrt{n}}+\frac{2}{\sqrt{n}}
$$

Proof: For any integer $m>0$ by the Reflection Principle we have

$$
\mathbf{P}\left(\bar{Y}_{n} \geq m\right) \geq 2 \mathbf{P}\left(Y_{n}>m\right)
$$

since with every path $\omega$ such that $Y_{n}(\omega)>m$, we can associate two paths for which $\bar{Y}_{n} \geq m$ : one path is itself, and the other path $\omega^{\prime}$ is identical to $\omega$ except between times $i$ and $n$, where $i$ is the first time $S_{i}=m !$ between times $i$ and $n, \omega^{\prime}$ is the reflection of $\omega$ through the line $y=m$. Thus,

$$
\begin{aligned}
\mathbf{P}\left[\bar{Y}_{n}>[a\rfloor\right] & \geq 2 \mathbf{P}\left[Y_{n}>\lfloor a\rfloor\right] \geq 2 \mathbf{P}\left[\frac{Y_{n}}{\sqrt{n}}>\frac{a}{\sqrt{n}}\right]=2\left[1-\mathbf{P}\left[\frac{Y_{n}}{\sqrt{n}} \leq \frac{a}{\sqrt{n}}\right]\right] \\
& \geq 2\left[1-N\left(\frac{a}{\sqrt{n}}\right)-\frac{1}{\sqrt{n}}\right] \quad \text { (Berry-Essen Theorem) } \\
& \geq 1-\sqrt{\frac{2}{\pi}} \frac{a}{\sqrt{n}}-\frac{2}{\sqrt{n}} \quad\left(\text { (since } N(x) \leq \frac{1}{2}+x \text { for } x>0\right) .
\end{aligned}
$$

The following is a straightforward application of this theorem.

Corollary 6 (Averages in an undrifted random walk.) Consider the binomialstock price process with $p=$ $\frac{1}{2}$. starting with price $S_{0}$ at time 0 , and let $K$ be the strike price in the Asian option. Let

$$
\mathbf{P}_{e}(n) \triangleq \sqrt{\frac{2}{\pi}} \frac{\log _{u}\left(n K / S_{0}\right)}{\sqrt{n}}+\frac{1}{\sqrt{n}}
$$

Then for $n>S_{0} / K$, with probability at least $1-\mathbf{P}_{e}(n)$. the maximum stock price on a path is at least $n K$. and in particular $\mu_{n} \geq K$.

Proof: See the Appendix. 


\section{Approximating an $n$-period American put with a perpetual put}

Recall that a Markovian American option (MAO) is one whose payoff is given by $\left(c_{k}=g\left(S_{k}\right)\right.$ for some function $g$. The dynamic programming algorithm (hased on the backward recursion (5)) for pricing a MAO requires $O\left(n^{2} i\right.$ time. On the other hand, the value $l$ of some perpetual Markovian American options (PMAO). such as perpetual American puts. can be computed in closed form in only $O(1)$ time. It is therefore of interest to investigate how well the value of a PMAO approximates the value of an otherwise identical MAO. In this section we first show a general formula bounding the difference between a PMAO and an otherwise identical MAO, and then apply it to the case of American puts. It is not hard to show (see Hull [16]) that under the pricing model of this paper, it is never optimal to exercise an American call before expiration. An American call is therefore equivalent to a European call and can be priced in $O(n)$ time. Thus much research has focused on devising fast pricing methods for American puts $[19,12,6]$. It is known [9] that the value of an American perpetual put can be computed in $O(1)$ time. In this section we investigate the difference between an American put and an otherwise equivalent American perpetual put.

Recall that $\mathcal{T}_{0}$ is the set of stopping times $\tau$ such that $\tau \geq 0$ almost surely. The value of a $n$-period MAO with initial (non-random) stock price $S_{0}$ is denoted by $l^{n}$. The value of a PMAO with initial stock price $S_{4}$ is denoted by $V$. From Section 1.3 we have the following formulas:

$$
\begin{aligned}
V^{n} & =\max _{\tau \in \mathcal{T}_{0}} \mathbf{E}\left[(1+r)^{-\tau \wedge n} g\left(S_{\tau \wedge n}\right)\right] \\
V & =\max _{\tau \in \tau_{0}} \mathbf{E}\left[(1+r)^{-\tau} g\left(S_{\tau}\right)\right],
\end{aligned}
$$

where $x \wedge y=\min \{x, y\}$. In Appendix A. 2 we prove the following lemma bounding the difference between a MAO and an otherwise identical PMAO with payoff $G_{k}=g\left(S_{k}\right)$.

Lemma 7 Let $\tau^{*}$ be a stopping time such that

$$
V=\mathbf{E}\left[(1+r)^{-\tau^{*}} g\left(S_{\tau^{*}}\right)\right] .
$$

Define $\lrcorner^{n}$ as follows:

$$
\Delta^{n}=\mathbf{E}\left[1_{\left\{\tau^{*}>n\right\}}\left((1+r)^{-\tau^{*}} g\left(S_{\tau^{*}}\right\}-(1+r)^{-n} g\left(S_{n}\right)\right)\right] .
$$

Then

$$
V-V^{n} \leq \Delta^{n}
$$

Now consider an American perpetual put. The payoff function in this case is given by $g\left(S_{k}\right)=\left(K^{*}-\right.$ $\left.S_{k}\right)^{+}$, where $K$ is the strike price. The following Lemma is known [9].

Lemma 8 For any integer $j \in \mathbf{Z}$, let $\tau_{j}$ denote the stopping time

$$
\tau_{j}=\min \left\{k: S_{k} \leq S_{0} u^{j}\right\}=\min \left\{k: Y_{k} \leq j\right\} .
$$

Given an American put with strike price $K$, there exists an integer $s \geq 0$ such that $\tau_{-s}$ achieves the max in Eq.(7):

$$
V=\mathbf{E}\left[(1+r)^{-\tau_{-s}} g\left(S_{\tau_{-s}}\right)\right]=\left(K-S_{0} u^{-s}\right) \mathbf{E}\left[(1+r)^{-\tau_{-s}}\right]
$$

The last expression in (8) can be computed in closed form [9]. In the following we assume that $s$ denotes the non-negative integer of Lemma 8 . Let $E_{k:}^{n}$ denote the event $\left\{S_{r_{i}}=S_{0} u^{k}, \tau_{-s}>n\right\}$, and let $P_{k}^{n} \triangleq \mathbf{P}\left[E_{k i}^{n}\right]$. We now upper bound the difference between an $n$-period American put and the corresponding perpetual put. 
Theorem 9 If $V^{-r}$ is the value of an n-period American put and $\mathrm{V}$ is the value of an otherwise identical American perpetual put (APP), then

$$
V-1^{n} \leq(1+r)^{-n} \sum_{k=-s+1}^{n} P_{k}^{n}\left(\left(K-S_{0} u^{-s}\right) a^{k+s}-\left(K-S_{0} u^{k}\right)^{+}\right) .
$$

where $\alpha \hat{\triangleq}\left[(1+r)^{--i}\right]$, and

$$
P_{k}^{n}=p^{\frac{n+k}{2}}(1-p)^{\frac{n-k}{2}}\left(\left(\begin{array}{c}
n \\
\frac{n+k}{2}
\end{array}\right)-\left(\begin{array}{c}
n \\
\frac{n+k+2 . s}{2}
\end{array}\right)\right) .
$$

Proof: The proof, given in Appendix A.2, uses Lemma 7 and the Reflection Principle.

We now obtain an asymptotic error bound from this Theorem. Recall that $\underline{Y}_{n} \triangleq \min _{0 \leq i \leq n} Y_{i}$, where $Y_{i}$ is as before the $i$ 'th partial sum in the random walk underlying the model. Noting that $\left(K-S_{0} u^{-s}\right) \leq K^{\text {. }}$ and $\left(K-S_{0} u^{k}\right)^{+} \geq 0$, we have for $p=\frac{1}{2}$ :

$$
\begin{aligned}
V-V^{n} & \leq K(1+r)^{-n} \sum_{k=-s+1}^{n} P_{k}^{n} \\
& =K(1+r)^{-n} \mathbf{P}\left[\underline{Y}_{n}>-s\right]=K(1+r)^{-n} \mathbf{P}\left[\bar{Y}_{n}<s\right] \\
& \leq K(1+r)^{-n}\left(\sqrt{\frac{2}{\pi}} \frac{s}{\sqrt{n}}+\frac{2}{\sqrt{n}}\right)=K(1+r)^{-n} O\left(\frac{1}{\sqrt{n}}\right) .
\end{aligned}
$$

Since the value $\mathbf{P}\left[\underline{Y}_{n}>-s\right]$ is a non-decreasing function of $p$, the above error bound also applies for $p<\frac{1}{2}$. We leave the asymptotic error analysis for $p>\frac{1}{2}$ as an open problem.

\section{Further research}

Some problems left open in this paper are: (a) obtaining a more accurate error bound for the Asian call approximation for $p<\frac{1}{2}$ (Section 4), and for the American put for $p>\frac{1}{2}$ (Section 5); (b) establishing the hardness of the pricing of a (European style) Asian option.

There are plenty of research directions to pursue in option pricing. We mention a few here. One important problem is the approximate pricing of American style Asian options, i.e., those that can be exercised at any time up to expiration. We saw in Sections 1.3 and 3 that certain American options can be priced in polynomial-time (in the maturity $n$ ) using dynamic programming. Devising fast (say linear-time) approximate algorithms for such options would be a significant contribution to quantitative finance. Another problem is option pricing with time-varying interest rate $r$ and time-varying up-factor $u$. Finally, we mention that Arbitrage Pricing Theory depends on the ability to perfectly hedge the option being priced. Soner, Shreve and Cvitanic [23] have shown for the continuous-time setting that when proportional transaction costs (such as broker commissions) are present, perfect hedging becomes impossible, and the pricing formulas of Section 1.3 no longer hold. An intriguing problem is therefore to develop a satisfactory pricing theory in the presence of transaction costs. Some initial work in this direction for simple calls and puts has been done $[1,5]$. 


\section{References}

[1] B. Bensaid. J. Lesne, H. Pages. and J. Scheinkman. Derivative asset pricing with transaction cost. Wath. Fin., 2(2):63-86, April 199?.

[2] F. Black and M. Scholes. The pricing of options and corporate liabilities. J. Political Economy, 81:637654.1973.

[3] L. Bouaziz. E. Briys, and M. Crouhy. The pricing of forward-starting asian options. J. Banking and Finunce, pages 823-839, 1994.

[4] P. Boyle. Options: A monte carlo approach. J. Financial Economics, 4:323-338, 1977.

[5] T. Boyle and T. Vorst. Option replication in discrete time with trasaction costs. J. Finance, XLVII(1):271-93, March 1992.

[6] M. Brennan and E. Schwartz. The valuation of american put options. Journal of Finance, 32, 1977.

[7] M. Brennan and E. Schwartz. Finite difference methods and jump pr :asses arising in the pricing of contingent claims. J. Fin. and Quant. Analysis, 13:462-474, 1978.

[8] J. Cox, S. Ross, and M. Rubinstein. Option pricing: A simplified approach. J. Financial Economics. 7:229-264, 1979.

[9] D. Duffie. Security Markets: Stochastic Models. Academic Press, 1988.

[10] R. Durrett. Probabilit:: Theory and Examples. Duxbury Press, 2nd edition, 1995.

[11] H. Geman and M. Yor. Bessel processes, asian options and perpetuities. In FORC Conference. Warrick. UK, 1992.

[12] R. Geske and H. Johnson. The american put valued analytically. Journal of Finance, 39:1511-24, 1984.

[13] M. Goldman, H. Sozin, and M. Gatto. Path dependent options: buy at the low, sell at the high. $J$. Finance, pages 1111-1128, 1979.

[14] B. Heenk, A. Kemna, and A. Vorst. Asian options on oil spreads. Review of Futures Markets, 9(3):510$528,1990$.

[15] W. Hoeffding. Probability inequalities for sums of bounded random variables. J. Am. Stat. Assoc., 58:13-30, 1963.

[16] J. Hull. Options, Futures, and Other Derivative Securities. Prentice Hall, 2nd edition, 1993.

[17] J. Hull and A. White. Valuing derivative securities using the explicit finite difference method. J. Fin. and Quant. Analysis, 25:87-100, 1990.

[18] J. Hull and A. White. Efficient procedures for valuing european and american path-dependent options. Journal of Derivatives, 1:21-31, 1993.

[19] H. Johnson. An analytic approximation to the american put price. J. Fin. and Quant. Analysis, 18:141$48,1983$.

[20] E. Manoukian. Modern Concepts and Theorems of Mathematical Statistics. Springer-Verlag, 1985. 
[21] H. Niederreiter. Random Number Generation and Quasi-Monte Carlo Methods. SIAM, 1992.

[22] R. Rubinstein. Simulation and the Monte Carlo Method. John Wiley and Sons, 1981.

[23] H. Soner, S. Shreve, and J. Cvitanic. There is no non-trivial hedging portfolio for option pricing with transaction costs. Annals of Applied Probability. 5(2):327-55, 1995.

[24] D. Stroock and S. Varadhan. Multidimensionnal Diffusion Processes. Springer-Verlag, 1979.

[25] S. Turnbull and L. Wakeman. A quick algorithm for pricing european average options. I. Financial and Quantitative Analysis, 26(3):377-390, 1991.

[26] D. Williams. Probability with Martingales. Cambridge Univ. Press, 1992.

[27] P. Wilmott, J. DeWynne, and S. Howison. Option pricing: mathematical models and computation. Oxford Financial Press, 1993.

[28] P. Zhang. Flexible arithmetic asian options. Journal of Derivatives, pages 53-63, Spring 1995.

\section{A APPENDIX}

\section{A.1 Standard results assumed in the paper}

\section{A.1.1 Random walks}

Theorem 10 (Hoeffding[15]) Let $X_{1}, X_{2}, \ldots, X_{n}$ be independent, identically-distributed (i.i.d.) random variables whose values lie in the interval [-1,1], and let $Y_{n}=X_{1}+X_{2}+\ldots+X_{n}$. Then for $a>0$ the following holds:

$$
\begin{aligned}
& \mathbf{P}\left[Y_{n}-\mathbf{E} Y_{n}<-a\right]<e^{-\frac{a^{2}}{2 n}} \\
& \mathbf{P}\left[Y_{n}-\mathbf{E} Y_{n}>+a\right]<e^{-\frac{a^{2}}{2 n}}
\end{aligned}
$$

In particular, for the random walk with drift $p$, we have the iid random variables $X_{1}, X_{2}, \ldots, X_{n}$ where $\mathbf{P}\left[X_{i}=+1\right]=p$ and $\mathbf{P}\left[X_{i}=-1\right]=1-p$, and so $\mathbf{E} Y_{i}=(2 p-1) i$.

Theorem 11 (Berry-Essen $[10,20]$ ) Let $X_{1}, X_{2}, \ldots, X_{n}$ be i.i.d. with $\mathbf{E} X_{i}=0, \mathbf{E} X_{i}^{2}=\sigma^{2}$, and $\mathbf{E}\left|\mathbf{X}_{i}\right|^{3}=$ $\xi$. If $F_{n}(x)$ is the distribution of $\frac{\left(X_{1}+X_{2}+\ldots+X_{n}\right)}{\sigma \sqrt{n}}$ and $N(x)$ is the standard normal distribution, then

$$
\left|F_{n}(x)-N(x)\right| \leq \frac{\xi}{\sigma^{3} \sqrt{n}}
$$

Theorem 12 ((Reflection Principle)[10]) Imagine drawing the paths of the random walk on the $x-y$ plane as follows: for each $i=0.1 \ldots$ draw an edge from $\left(i, Y_{i}\right)$ to $\left(i+1, Y_{i+1}\right)$, where $Y_{i}$ is the $i$ th partial sum. If $T, T^{\prime}$ are positive integers, the number of paths from $(0, T)$ to $\left(n, T^{\prime}\right)$ that touch or cross the $x$-axis is equal to the number of paths from $(0,-T)$ to $\left(n, T^{\prime}\right)$.

Stopping time[26] Let $\Omega$ be the sample space of all possible coin-toss paths $\omega$ defined in Section 1.1. A stopping time is a random variable $\tau: \Omega \rightarrow\{0.1 .2 \ldots, n\} \cup\{\infty\}$ with the property that for each $k=$ $0,1, \ldots, n . \infty$, the set $\{\tau=k\}$ belongs to the $\sigma$-algebra $\mathcal{F}_{k}$. This means that membership in the set $\{\tau=k\}$ depends only on the first $k$ coin tosses of $\omega$. Informally, a stopping time can be thought of as a "decision rule" of when to "stop" the coin-toss sequence (or the random walk). 


\section{A.2 Proofs of some results}

\section{Proof of Theorem 3}

Proof: Suppose $p>\frac{1}{2}$. and let $E_{k}$. denote the event $\left\{Y_{i}<1\right\}$. Then we have

$$
\begin{aligned}
\mathbf{P}\left[E_{k}\right] & =\mathbf{P}\left[\xi_{k}<a\right] \\
& =\mathbf{P}\left[\mathbf{E} Y_{k}-\xi_{k}>\mathbf{E} Y_{k}-a\right] .
\end{aligned}
$$

and since $\mathrm{E} \xi_{k}=(2 p-1) k=3 k \geq 3 m>2 a>a$, by Hoeffding bounds (Appendix, eq. $(\mathrm{H})$ ):

$$
\begin{aligned}
& \leq \exp \left\{-\frac{1}{2 k}\left(3^{2} k^{2}-2 k 3 a+a^{2}\right)\right\} \\
& =\exp \left\{3 a-3^{2} k / 2\right\} \cdot \underbrace{\epsilon^{-\frac{1}{2} \pi^{2} / k}}_{\leq 1} \\
& \leq \exp \left\{3 a-3^{2} k / 2\right\} .
\end{aligned}
$$

Therefore.

$$
\begin{aligned}
\sum_{i=m}^{n} \mathbf{P}\left[E_{k}\right] & \leq \epsilon^{3 a} \int_{m}^{x} e^{-3^{2} x / 2} d x \\
& =\frac{2}{3^{2}} \exp \left\{3 a-3^{2} m / 2\right\}
\end{aligned}
$$

The proof for $p<\frac{1}{2}$ is exactly analogous.

\section{Proof of Corollary 4}

Proof: Let $X_{1}, X_{2} \ldots \ldots X_{r_{i}}$ denote the random walk underlying the binomial process, and let $Y_{i}$ be the $i$ th partial sum as in Theorem 3. Thus the stock price after $i$ coin tosses is $S_{0} u^{Y_{i}}$.

Case 1: $p>\frac{1}{2}$. Applying Theorem 3 with $m=\left\lfloor n / 2\left\lfloor\right.\right.$ and $a=\log _{u}\left(2 K / S_{0}\right)$, we see that with probability at least $1-\mathbf{P}_{3}(n)$, we have that $Y_{i} \geq \log _{u}\left(2 K / S_{0}\right)$ for every $i \geq n / 2$, or in other words, the stock price $S_{i}$ for $i \geq n / 2$ is at least $2 K$, in which case average over all stock prices $S_{i}$ is at least $K$.

Case 2: $p<\frac{1}{2}$. Applying Theorem 3 with $m$ as in the statement of the present theorem and $a=\log _{u} n$ we see that with probability at least $1-\mathbf{P}_{\ell}(c, n)$, we have that $Y_{i} \leq-\log _{u} n$ for every $i \geq m$, or in other words. every stock price $S_{i}$ for $i \geq m$ is at most $S_{0} / n$. In such an event, the contribution of each stock price after $S_{i} . i \geq ! \quad 0 \mu_{n}$ is no more than $S_{0} / n^{2}$, so that the "error" in estimating $\mu_{n}$ by $T_{m} / n$ is at most $S_{0} / n$.

\section{Proof of Corollary 6}

Proof: Let $Y_{1}, Y_{2} \ldots \ldots Y_{n}$ be the random walk underlying the binomial model, and let $Y_{i}$ be the $i$ 'th partial sum as betore. Applying Theorem 5 with $a=\log _{u}\left(n K / S_{0}\right)$ we see that with probability at least $1-\mathbf{P}_{\varepsilon}(n)$ the highest $Y_{i}$ is at least $a$, so that the highest stock price is at least $S_{0} u^{a}=n K$. In this case the average stock price over the path is at least $K$. 


\section{Proof of Lemma 7}

Proof: We have

$$
\begin{aligned}
& V^{n} \geq \mathbf{E}\left[(1+r)^{-r^{*} \wedge n} g\left(S_{r * * r}\right)\right] \\
& =\mathbf{E}\left[1_{r^{*}>r_{i}}(1+r)^{-*} g\left(S_{r}\right)\right]+\mathbf{E}\left[1_{\tau^{*}<r_{i}}(1+r)^{-r^{*}} g\left(S_{\tau^{*}}\right)\right] \text {. } \\
& V=\mathbf{E}\left[1_{r^{*}>r}(1+r)^{-\cdot *} g\left(S_{-*}\right)\right]+\mathbf{E}\left[1_{\tau_{*}<n}(1+r)^{-*} g\left(S_{-*}\right)\right] \text {. }
\end{aligned}
$$

and the lemma follows.

\section{Proof of Theorem 9}

Proof: We use Lemma 7, with $\tau^{*}=\tau_{-s}$ :

$$
\begin{aligned}
\mathbf{E}\left[\mathbf{1}_{\tau^{*}>n}(1+r)^{-\tau^{*}} g\left(S_{\tau^{*}}\right)\right] & =\sum_{k=-s+1}^{n} P_{k}^{n} \mathbf{E}\left[(1+r)^{-\tau^{*}} g\left(S_{\tau^{*}}\right) \mid E_{k}^{n}\right] \\
& =(1+r)^{-n} \sum_{k=-s+1}^{n} P_{k}^{n} g\left(S_{\tau_{-s}}\right) \mathbf{E}\left[(1+r)^{-\tau_{-s-k}}\right] \\
& =(1+r)^{-n} \sum_{k=-s+1}^{n} P_{k}^{n}\left(K-S_{0} u^{-s}\right) \alpha^{k+s} . \\
\mathbf{E}\left[\mathbf{1}_{\tau^{*}>n}(1+r)^{-n} g\left(S_{n}\right)\right] & =(1+r)^{-n} \sum_{k=-s+1}^{n} P_{k}^{n}\left(K-S_{0} u^{k}\right)^{+} .
\end{aligned}
$$

We are using the fact that

$$
E\left[(1+r)^{-\tau-k}\right]=\left(E\left[(1+r)^{-\tau-1}\right]\right)^{k}(\text { for } k \geq 1) .
$$

The expression for $P_{k}^{n}$ can be derived using the Reflection Principle (see the Appendix).

\section{A.3 A path-clustering approximation for Asian options}

We present here an $O\left(n^{3}\right)$-time approximation algorithm for an Asian Call for the case $p \geq \frac{1}{2}$ that in practice performs significantly better than the algorithm presented above. We leave the error analysis of this algorithm as an open problem.

Note that

$$
\begin{aligned}
V & =(1+r)^{-n} \mathbf{E}\left(\mu_{n}-K^{-}\right)^{+} \\
& =(1+r)^{-n} \sum_{\omega \in\{H . T\}^{n}} \mathbf{P}(\omega)\left(\mu_{n}(\omega)-K\right)^{+} \\
& =(1+r)^{-n} \sum_{k=0}^{n} \sum_{H(\omega)=k} \mathbf{P}(\omega)\left(\mu_{n}(\omega)-K\right)^{+} \\
& =(1+r)^{-n} \sum_{k=0}^{n} p^{k} q^{n-k} \sum_{H(\omega)=k}\left(\mu_{n}(\omega)-K\right)^{+},
\end{aligned}
$$

where $H(\omega)$ denotes the number of $H$ 's in the path $\omega$. 
For each $k$ we approximate the sum in (9) as follows:

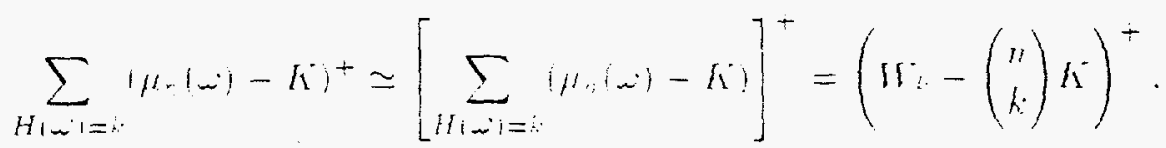

where

$$
W_{i} \doteq \sum_{H(-i)=k} \mu_{r:}(\omega)
$$

Thus our approximation for $F$ is

$$
(1+r)^{-n} \sum_{k=0}^{n} p^{k} q^{n-k}\left(w_{k}-\left(\begin{array}{l}
n \\
k
\end{array}\right) K\right)^{+}
$$

Note that $W_{k} /\left(\begin{array}{c}r_{i} \\ k\end{array}\right)$ is the expected value of $\mu_{n}$ over the "cluster" of paths that have exactly $k H$ 's. Thus our approximation is similar in spirit to computing $\left(\mathbf{E} / i_{r_{2}}-K\right)^{\top}$. The difference is that instead of computing the expected value of $\mu_{n}(\omega)$ over all paths $\omega$ and then thresholding with $k^{-}$(i.e. computing $\left(.-K^{-}\right)^{+}$), we compute the expected value of $\mu_{n}(\omega)$ for each cluster and apply the threshold $(.-k)^{+}$to each cluster.

We now show that $W_{k}$ can be computed in polynomial time. For $p \leq n . h \leq p$, we say that a path

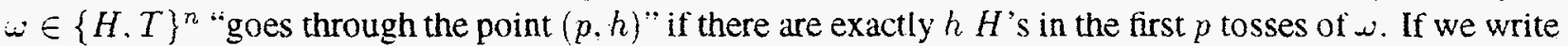
down the expression for $W_{k}$ we see that the stock price at point $(p, h)$ (which is $S_{0} u^{2 h-p}$ ) gets multiplied by a factor

$$
\frac{\left(\begin{array}{l}
p \\
h
\end{array}\right)\left(\begin{array}{c}
n-p \\
k-h
\end{array}\right)}{(n+1)}
$$

so that $H_{k}$ may be written

$$
W_{k}=\frac{S_{0}}{(n+1)} \sum_{p=0}^{n} \sum_{h=0}^{p}\left(\begin{array}{l}
p \\
h
\end{array}\right)\left(\begin{array}{l}
n-p \\
k-h
\end{array}\right) u^{2 h-n}
$$

It is easy to see that this new approximation algorithm takes $O\left(n^{3}\right)$ time. 


\section{B Summary of Notation}

For each symbol, we mention the page where it is defined, and give a brief definition.

\begin{tabular}{|c|c|c|}
\hline Symbol & Page & Brief definition \\
\hline 3 & 6 & $|2 p-1|$ \\
\hline $\mathcal{F}_{k}$ & 2 & $\sigma$-field generated by the first $k$ coin-tosses. \\
\hline$G_{k}$ & 3 & The payoff from an option exericised at time $k$. \\
\hline$k$ & 3 & Strike price of an option. \\
\hline$\mu_{n}$ & 3 & Average stock price from time 1 to time $n, T_{n} / n$ \\
\hline$n$ & 2 & Maturity of the option. (Infinite for perpetual options) \\
\hline$\omega$ & 2 & A coin-toss sequence $\omega_{1}, \omega_{2} \ldots, \omega_{n}$ of length $n$ \\
\hline$\Omega$ & 2 & The sample space of all coin-toss sequences of length $n$. \\
\hline$p$ & 2 & Up-tick probability, i.e., probability of occurrence of $H$. \\
\hline $\mathbf{P}_{\ell}(c, n)$ & 7 & Probability bound defined in Corollary 4 , for the case $p<\frac{1}{2}$. \\
\hline $\mathbf{P}_{g}(n)$ & 7 & Probability bound defined in Corollary 4 , for the case $p>\frac{1}{2}$. \\
\hline $\mathbf{P}_{e}(n)$ & 8 & Probability bound defined in Corollary 6 , for the case $p=\frac{1}{2}$. \\
\hline$S_{k}$ & 2 & Stock price at time $k,=S_{0} u^{Y_{k+1}}$ \\
\hline $\bar{S}_{k}$ & 2 & $\max _{0 \leq i \leq k} S_{i}$ \\
\hline$T_{k}$ & 2 & $T_{k}=\sum_{i=1}^{k} S_{i}$ \\
\hline$\tau$ & 4 & Generic stopping time. \\
\hline$T_{j}$ & 9 & The specific stopping time $\min \left\{k: Y_{k}=j\right\}$ \\
\hline $\mathcal{T}_{k}$ & 4 & Class of stopping times $\tau$ such that $k \leq \tau \leq n$. \\
\hline$u$ & 2 & The up-factor. \\
\hline$V$ & 4 & Value of the option under consideration. \\
\hline & 9 & In Section 5 this is the value of an American perpetual put. \\
\hline$V^{n}$ & 9 & Value of an $n$-period American put. \\
\hline$X_{k}$ & 2 & Random variable: $X_{k}(\omega)=1$ if $\omega_{k}=H$ and $X_{k}(\omega)=-1$ otherwise. \\
\hline$Y_{k}$ & 2 & $Y_{k}=\sum_{i=1}^{k} X_{i} ; Y_{0}=0$ \\
\hline $\bar{Y}_{n}$ & 2 & $\max _{0 \leq i \leq n} Y_{i}$ \\
\hline$\underline{Y}_{n}$ & 2 & $\min _{0 \leq i \leq n} Y_{i}$ \\
\hline$x \wedge y$ & 9 & $\min \{\bar{x}, y\}$ \\
\hline$x^{+}$ & 3 & $\max \{x, 0\}$ \\
\hline
\end{tabular}


